\title{
Probabilistic analysis of the face stability of circular tunnels
}

\author{
Guilhem Mollon ${ }^{1}$, Daniel Dias ${ }^{2}$ and Abdul-Hamid Soubra ${ }^{3}$, M. ASCE \\ ${ }^{1}$ PhD Student, INSA Lyon, LGCIE Site Coulomb 3, Géotechnique, Bât. J.C.A. Coulomb, Domaine \\ scientifique de la Doua, 69621 Villeurbanne cedex, France. E-mail: Guilhem.Mollon@insa-lyon.fr \\ 2 Associate Professor, INSA Lyon, LGCIE Site Coulomb 3, Géotechnique, Bât. J.C.A. Coulomb, \\ Domaine scientifique de la Doua, 69621 Villeurbanne cedex, France. Email: Daniel.Dias@insa- \\ lyon.fr \\ ${ }^{3}$ Professor, University of Nantes, GeM, UMR CNRS 6183, Bd. de l'université, BP 152, 44603 Saint- \\ Nazaire cedex, France
}

\begin{abstract}
This paper presents a reliability-based approach for the threedimensional analysis of the face stability of a shallow circular tunnel driven by a pressurized shield. Only the collapse failure mode of the ultimate limit state is studied. The deterministic model is based on the upper-bound method of the limit analysis theory. The random variables used are the soil shear strength parameters. The Hasofer-Lind reliability index and the failure probability were determined. A sensitivity analysis was also performed. It was shown that (i) the assumption of negative correlation between the soil shear strength parameters gives a greater reliability of the tunnel face against collapse, with respect to the hypothesis of uncorrelated variables, (ii) the failure probability is much more influenced by the coefficient of variation of the angle of internal friction than that of the cohesion and (iii) when no correlation between shear strength parameters is considered, a more spread out CDF of the tunnel pressure was obtained in comparison to the case of correlated shear strength parameters.
\end{abstract}

\section{INTRODUCTION}

Over the past thirty years, tunnelling in a frictional and/or cohesive soil has been possible due to recent technological advances including the pressurized shield. Face stability analysis of shallow circular tunnels driven by the pressurized shield is of major importance. The tunnel face pressure must avoid both the collapse (active failure) and the blow-out (passive failure) of the soil mass nearby the tunnel face. In this paper, only the collapse mode of the ultimate limit state (ULS) is considered in the analysis. 
The face stability analysis is conducted based on a probabilistic approach. The soil shear strength parameters are modeled as random variables. The reliability-based analysis is more rational than the deterministic one since it takes into account the inherent uncertainty of the input variables. Nowadays, this is possible because of the improvement of our knowledge on the statistical properties of the soil (Phoon and Kulhawy 1999; Baecher and Christian 2003).

\section{OVERVIEW OF RELIABILITY CONCEPTS}

The reliability index is a measure of the safety that takes into account the inherent uncertainties of the input variables. A widely used reliability index is the Hasofer and Lind (1974) index. Its matrix formulation is (Ditlevsen 1981)

$$
\beta_{H L}=\min _{x \in F} \sqrt{(x-\mu)^{T} C^{-1}(x-\mu)}
$$

in which $x$ =vector representing the $n$ random variables; $\mu$ =vector of their mean values; $C=$ covariance matrix; and $F=$ failure region. The minimization of Eq. 1 is performed subject to the constraint $G(x) \leq 0$ where the limit state surface $G(x)=0$, separates the $n$ dimensional domain of random variables into two regions: A failure region $F$ represented by $G(x) \leq 0$ and a safe region given by $G(x)>0$.

Low and Tang (1997) have shown that the minimization of the reliability index is equivalent to find the smallest dispersion ellipsoid (or ellipse in the two-dimensional case) that is tangent to the limit state surface and whose centre is at the equivalent normal mean point of the random variables. This ellipsoid is called 'critical dispersion ellipsoid' and the ellipsoid corresponding to $\beta_{H L}=1$ in Eq. 1 without the min is named 'unit dispersion ellipsoid'. The ratio between the critical dispersion ellipsoid and the unit dispersion one is the Hasofer-Lind reliability index. When the random variables are non-normal and correlated, the optimisation approach uses the Rackwitz-Fiessler equivalent normal transformation without the need to diagonalize the correlation matrix as shown in Youssef Abdel Massih et al. (2008). The computations of the equivalent normal mean $\mu^{N}$ and equivalent normal standard deviation $\sigma^{N}$ for each trial design point are automatically found during the constrained optimization search. The method of computation of the reliability index using the concept of an expanding ellipse suggested by Low and Tang (1997) is used in this paper.

\section{RELIABILITY ANALYSIS OF A CIRCULAR TUNNEL AGAINST FACE COLLAPSE}

The aim of this paper is to perform a reliability analysis of a shallow circular tunnel against face collapse. The circular tunnel is driven by a pressurized shield in a $c-\varphi$ soil. The problem can be idealized as shown in Fig. 1 by considering a circular rigid tunnel of diameter $D$ driven under a depth of cover $C$. A surcharge $\sigma_{s}$ is applied at 
the ground surface and a constant retaining pressure $\sigma_{t}$ is applied to the tunnel face to simulate tunnelling under compressed air.

Due to uncertainties in soil shear strength parameters, the cohesion $c$, and the angle of internal friction $\varphi$ are considered as random variables. The performance function $\mathrm{G}$ used in the reliability analysis is defined as follows:

$$
G=\sigma_{t}-\sigma_{c}
$$

where $\sigma_{\mathrm{t}}$ is the applied pressure on the tunnel face, and $\sigma_{\mathrm{c}}$ is the collapse pressure. For the computation of the collapse pressure $\sigma_{c}$, a multiblok failure mechanism is proposed in the framework of the kinematical approach of limit analysis. The proposed MI mechanism allows the slip surface to develop more freely in comparison with the available mechanism given by Leca and Dormieux (1990). It is composed of several truncated rigid cones with circular cross-sections and with opening angles equal to $2 \varphi$. Each cone is the mirror image of the adjacent cone with respect to the normal plane to the surface separating these cones. This is a necessary condition to assure the same elliptical contact area between adjacent cones. The upper rigid cone will or will not intersect the ground surface depending on the C/D value. It should be mentioned that $M I$ is a translational kinematically admissible failure mechanism. The different blocks of this mechanism move as rigid bodies. These rigid cones translate with velocities of different directions, which are collinear with the cones axes and make an angle $\varphi$ with the discontinuity surface. The velocity of each cone is determined by the condition that the relative velocity between the cones in contact has the direction that makes an angle $\varphi$ with the contact surface. The velocity hodograph is presented in Fig. 1. The present mechanism is completely defined by $n$ angular parameters $\alpha$ and $\beta_{i}(i=1, \ldots, n-1)$ where $n$ is the number of rigid blocks.
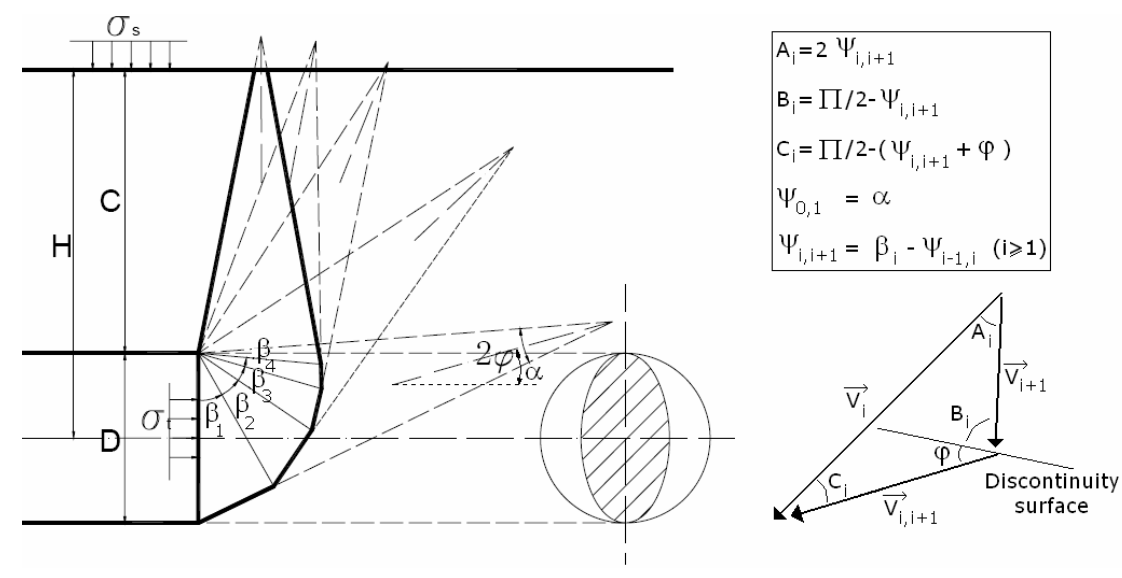

FIG. 1. Failure mechanism $M 1$

In the present paper, by the Low and Tang (1997) method, one literally sets up a tilted ellipsoid in the Excel spreadsheet and minimizes the dispersion ellipsoid subject to the constraint that it be tangent to the limit state surface. 


\section{NUMERICAL RESULTS}

For the $M 1$ mechanism, when the total rate of energy dissipation and the total rate of external work are equated, the collapse tunnel pressure $\sigma_{c}$ can be expressed as follows:

$\sigma_{c}=\gamma D N_{\gamma}+c N_{c}+\sigma_{s} N_{s}$

where $N_{\gamma}, N_{c}$, and $N_{s}$ are non-dimensional coefficients. They represent respectively the effect of soil weight, cohesion, and surcharge loading. The expressions of the different coefficients are given in Oberlé (1996). In Eq. 3, $\sigma_{c}$ depends not only on the mechanical and geometrical characteristics $c, \varphi$, and $C / D$, but also on the geometrical parameters of the failure mechanism $\alpha$ and $\beta_{i}$ $(i=1, \ldots, n-1)$. The deterministic collapse pressure $\sigma_{\mathrm{c}}$ was obtained by maximization of the Eq. 3 with respect to the $\alpha$ and $\beta_{i}$ angles. It was shown that the increase in the number of cones improves the solution. This improvement becomes insignificant (smaller than 1\%) for a number of blocks greater than five. Therefore, only five blocks were used in this paper. The present failure mechanism was shown to give a better (i.e. greater) solution than the available upper-bound solution given by Leca and Dormieux (1990). For more details on this comparison, the reader may refer to Soubra et al. (2008).

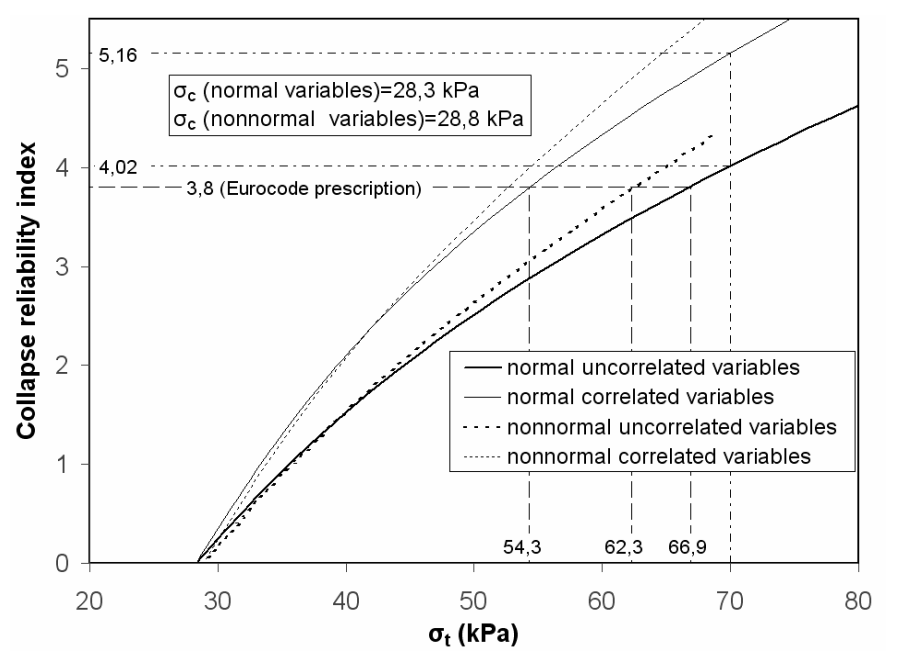

FIG. 2. Reliability index versus $\sigma_{t}$

The probabilistic numerical results presented in this paper consider the case of a circular tunnel with a diameter $\mathrm{D}=10 \mathrm{~m}$ and a cover $\mathrm{C}=10 \mathrm{~m}$ (i.e. $\mathrm{C} / \mathrm{D}=1$ ). The soil has a unit weight of $18 \mathrm{kN} / \mathrm{m}^{3}$. No surcharge loading $\left(\sigma_{s}=0\right)$ is considered in the analysis. For the probability distribution of the random variables, two cases are 
studied. In the first case, referred to as normal variables, $c$ and $\varphi$ are considered as normal variables. In the second case, referred to as nonnormal variables, $c$ is assumed to be lognormally distributed while $\varphi$ is assumed to be bounded and a beta distribution is used. The parameters of the beta distribution are determined from the mean value and standard deviation of $\varphi$ (Haldar and Mahadevan 2000). For both cases, correlated and uncorrelated variables are considered. In this paper, the illustrative values used for the statistical moments of the shear strength parameters and their coefficient of correlation $\rho_{c, \varphi}$ are as follows: $\mu_{c}=7 \mathrm{kPa}, \mu_{\varphi}=17^{\circ}$, $\operatorname{COV}_{c}=20 \%, \operatorname{COV}_{\varphi}=10 \%$ and $\rho_{c, \varphi}=-0.5$. The values of the COV and the negative correlation are taken from Phoon and Kulhawy (1999). The reliability index is determined by minimizing the quadratic form of Eq. 1 not only with respect to the random variables, but also with respect to the geometrical parameters of the failure mechanism $\left(\alpha, \beta_{i}\right)$ (cf. Youssef Abdel Massih et al. 2008). Thus, the minimization is performed with respect to 7 parameters $\left(\alpha, \beta_{i}, c, \varphi\right)$.

Table 1. Reliability index and design point

\begin{tabular}{c|c|cccccc}
\hline \multirow{5}{*}{ Normal variables } & $\begin{array}{c}\boldsymbol{\sigma}_{\boldsymbol{t}} \\
(\boldsymbol{k P a})\end{array}$ & \multicolumn{3}{|c}{ variables } & \multicolumn{3}{c}{ Correlated variables } \\
& $\boldsymbol{\beta}_{\mathrm{HL}}$ & $\mathbf{c}^{*}$ & $\boldsymbol{\varphi}^{*}$ & $\boldsymbol{\beta}_{\mathrm{HL}}$ & $\mathbf{c}^{*}$ & $\boldsymbol{\varphi}^{*}$ \\
\cline { 2 - 9 } & $\mathbf{2 8 . 3}$ & 0.00 & 7.00 & 17.00 & 0.00 & 7.00 & 17.00 \\
& $\mathbf{3 0}$ & 0.25 & 6.76 & 16.69 & 0.35 & 6.79 & 16.66 \\
& $\mathbf{3 5}$ & 0.93 & 6.18 & 15.78 & 1.30 & 6.38 & 15.57 \\
& $\mathbf{4 0}$ & 1.53 & 5.74 & 14.90 & 2.11 & 6.32 & 14.40 \\
& $\mathbf{5 0}$ & 2.51 & 5.19 & 13.33 & 3.35 & 6.54 & 12.37 \\
& $\mathbf{6 0}$ & 3.32 & 4.82 & 12.02 & 4.34 & 6.82 & 10.73 \\
& $\mathbf{7 0}$ & 4.02 & 4.53 & 10.87 & 5.16 & 7.11 & 9.34 \\
& $\mathbf{8 0}$ & 4.63 & 4.30 & 9.85 & 5.87 & 7.40 & 8.12 \\
& $\mathbf{1 0 0}$ & 5.69 & 3.95 & 8.07 & 7.08 & 7.94 & 6.05 \\
\hline & & $\boldsymbol{\beta}_{\mathrm{HL}}$ & $\mathbf{c}^{*}$ & $\boldsymbol{\varphi}^{*}$ & $\boldsymbol{\beta}_{\mathrm{HL}}$ & $\mathbf{c}^{*}$ & $\boldsymbol{\varphi}^{*}$ \\
\hline \multirow{5}{*}{ Nonnormal variables } & $\mathbf{2 8 . 8}$ & 0.00 & 6.86 & 16.97 & 0.00 & 6.86 & 16.97 \\
& $\mathbf{3 0}$ & 0.17 & 6.71 & 16.75 & 0.24 & 6.73 & 16.72 \\
& $\mathbf{3 5}$ & 0.89 & 6.19 & 15.77 & 1.23 & 6.40 & 15.56 \\
& $\mathbf{4 0}$ & 1.53 & 5.84 & 14.81 & 2.08 & 6.33 & 14.39 \\
& $\mathbf{5 0}$ & 2.63 & 5.42 & 13.17 & 3.47 & 6.33 & 12.51 \\
\hline
\end{tabular}

The collapse pressure was found equal to $\sigma_{c}=28.3 \mathrm{kPa}$ for normal variables and to $28.8 \mathrm{kPa}$ for nonnormal variables, using respectively the mean and equivalent mean values of the random variables. The corresponding reliability index is equal to zero. Fig. 2 and Table 1 present the Hasofer-Lind reliability index versus $\sigma_{t}$ for four combinations of normal, nonnormal, uncorrelated and correlated shear strength parameters. The reliability index corresponding to uncorrelated variables is smaller than the one of negatively correlated variables for both normal and nonnormal variables. One can conclude that assuming uncorrelated shear strength parameters is conservative in comparison to assuming negatively correlated parameters. 

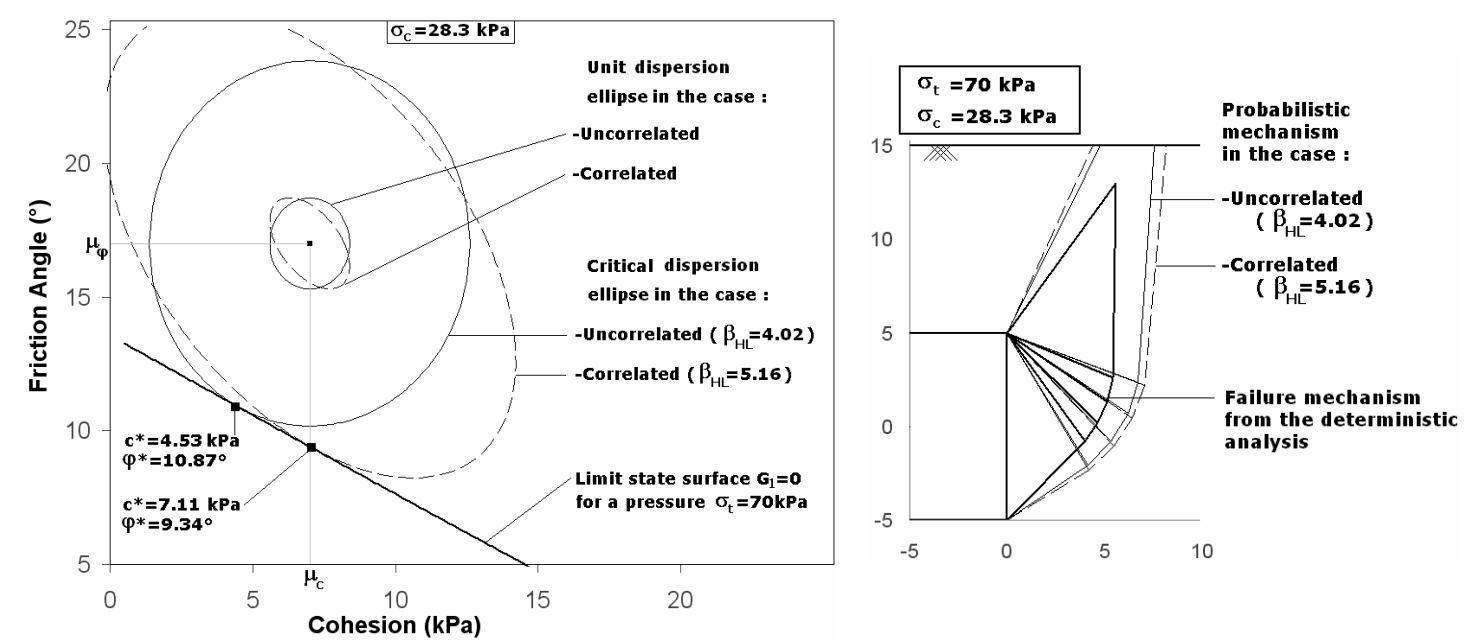

FIG. 3. Unit and critical dispersion ellipses for correlated and uncorrelated variables, and critical collapse mechanisms

For $\sigma_{t}=70 \mathrm{kPa}$ (Table 1), the reliability index for uncorrelated and correlated normal variables are respectively equal to 4.02 and 5.16. The corresponding most probable failure points obtained from the minimization procedure are found to be at $\left(c^{*}=4.53 \mathrm{kPa}, \varphi^{*}=10.87^{\circ}\right)$ and $\left(c^{*}=7.11 \mathrm{kPa}, \varphi^{*}=9.34^{\circ}\right)$. These are the points of tangency of the critical dispersion ellipses with the limit state surface. Fig. 3 provides graphical representation of the reliability analysis for both cases in the physical space of the random variables. One can easily see that negative correlation between shear strength parameters rotates the major axis of the ellipse from the vertical direction. The critical probabilistic failure mechanisms obtained for both uncorrelated and negatively correlated variables are also plotted in Fig. 3 using the values $c^{*}$ and $\varphi^{*}$ of the design points and the corresponding critical angular parameters of the failure mechanism. One can observe that the most probable failure mechanisms (i.e. the mechanisms plotted with the optimized angles at the design point) in the two cases are much more "extended" than the critical failure mechanism obtained in the deterministic analysis by optimization of the tunnel pressureThis is due to the fact that the probabilistic failure mechanisms correspond to a smaller value of $\varphi$.

Fig. 4 presents the $C D F \mathrm{~s}$ of the tunnel face pressure for normal, nonnormal, correlated and uncorrelated variables as given by FORM. When no correlation between shear strength parameters is considered, one can notice a more spread out CDF of the tunnel pressure (i.e., a higher coefficient of variation of this pressure) with respect to the case of correlated shear strength. Notice however that the chosen probability distribution (i.e., normal, lognormal, and beta distribution) does not significantly affect the values of the failure probability.

Fig. 5 presents the effect of the coefficient of variation of $c$ and $\varphi$ on the failure probability. A small change in the coefficient of variation of $\varphi$ highly affects the failure probability, but this failure probability is less sensitive to changes in the uncertainty of c. Thus, the failure probability is highly influenced by the coefficient of variation of $\varphi$. 


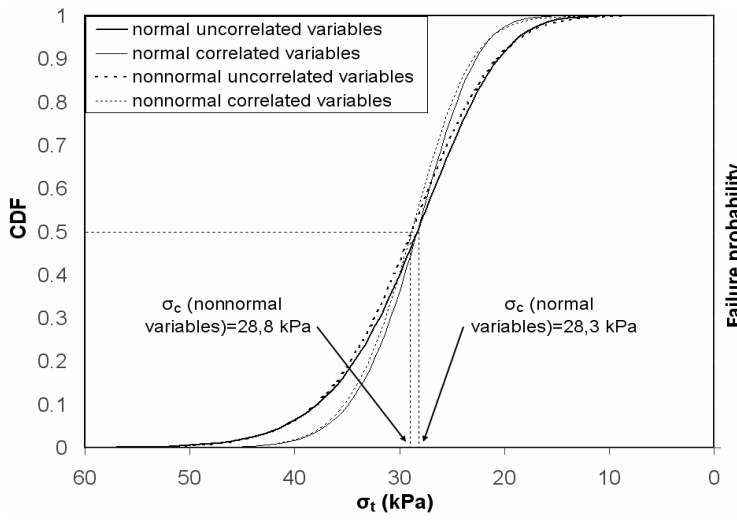

FIG. 4. CDFs of the tunnel face pressure

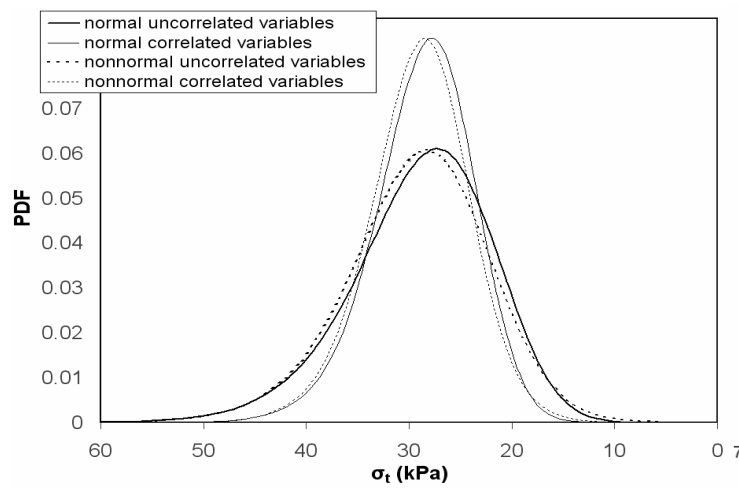

FIG. 6. PDFs of the tunnel face pressure

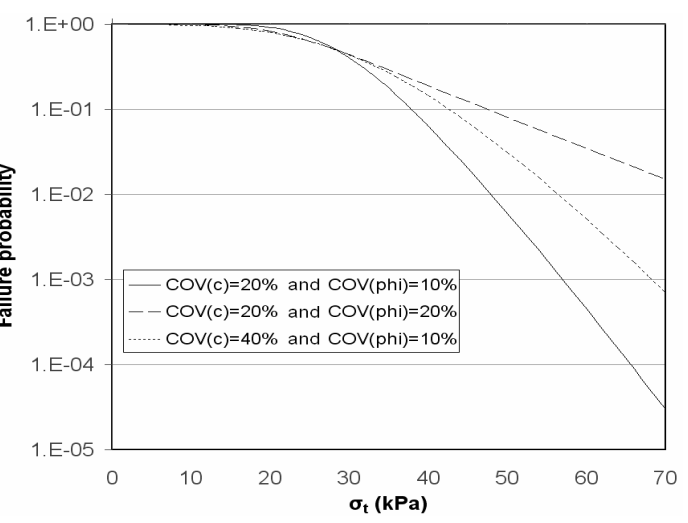

FIG. 5. Influence of the coefficients of variation of $c$ and $\varphi$

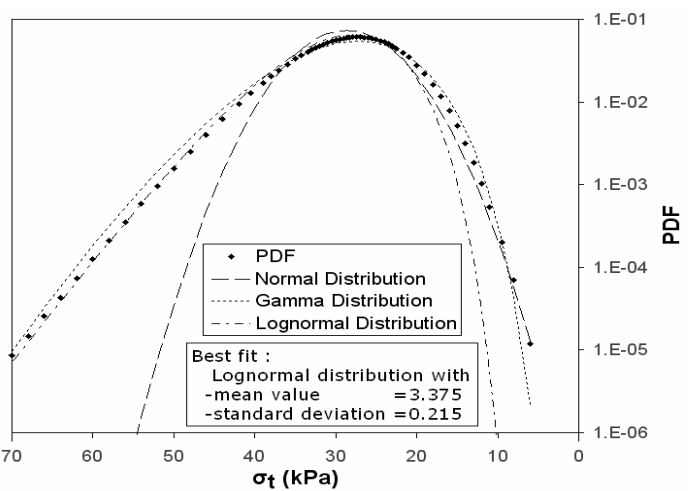

FIG. 7. Fit of the PDF of the tunnel pressure

Fig. 6 shows the PDFs corresponding to the CDFs given in Fig. 4. The PDFs were determined by numerical derivation of the CDFs. It can be seen that the results of normal and nonnormal variables are nearly similar. The correlation between the variables has on the contrary an important influence, making the probability density more significant around the deterministic value of the applied pressure. By fitting the PDF of the tunnel pressure to an empirical probability density function (Normal, Lognormal, Gamma) as shown in Fig. 7, it was found (after minimization of the sum of the relative errors between the values of the computed PDF and those of the empirical distribution) that the lognormal distribution is the one that best fits the computed PDF especially in the distribution tail (i.e. where $\sigma_{t}>2 \sigma_{c}$ ). It is then easy to use this lognormal distribution function to determine the failure probability for a given applied tunnel pressure.

\section{CONCLUSION}

A reliability-based analysis of the face stability of a shallow circular tunnel driven by a pressurized shield was performed. Only the collapse failure mode of the ultimate limit state was studied. A deterministic model based on the upper-bound method of limit analysis was used. The main conclusions of the paper are: 
- The assumption of uncorrelated shear strength parameters was found conservative (i.e., it gives a greater failure probability) in comparison to that of negatively correlated parameters; however, the type of the probability distribution does not significantly affect the values of the failure probability;

- The failure probability is more sensitive to $\varphi$ than to $c$. The greater the scatter in $\varphi$, the higher the failure probability. This means that the accurate determination of the uncertainties of $\varphi$ is important in obtaining reliable probabilistic results;

- When no correlation between shear strength parameters is considered, a more spread out CDF of the tunnel pressure was obtained in comparison to the case of correlated shear strength parameters;

- The distribution of the probability density function of the tunnel pressure was found very close to a lognormal distribution. This allows one to easily determine the failure probability against collapse for a given face pressure.

- In this study, the soil is considered as homogeneous in each simulation, and the variability is taken into account from one simulation to another. A next step would be to study the influence of a spatially varying field of the strength parameters, to be as close as possible to a real soil.

\section{REFERENCES}

Baecher, G.B., and Christian, J.T. (2003). Reliability and statistics in geotechnical engineering, Wiley.

Ditlevsen, O. (1981). Uncertainty modelling: With applications to multidimensional civil engineering systems, McGraw-Hill, New York.

Haldar, A., and Mahadevan, S. (2000). Probability, reliability and statistical methods in engineering design, Wiley, New York.

Hasofer, A. M., and Lind, N. C. (1974). "Exact and invariant second-moment code format." J. of Engrg. Mech. Div., 100 (1): 111-121.

Leca, E., and Dormieux, L. (1990). "Upper and lower bound solutions for the face stability of shallow circular tunnels in frictional material." Géotechnique, 40 (4): 581-606.

Low, B. K., and Tang, W. H. (1997). "Efficient reliability evaluation using spreadsheet." J. Eng. Mech., 123 (7): 749-752.

Oberlé, S. (1996). Application de la méthode cinématique à l'étude de la stabilité d'un front de taille de tunnel, Final Project, ENSAIS, France, in French.

Phoon, K.-K., and Kulhawy, F. H. (1999). "Evaluation of geotechnical property variability." Can. Geotech. J., 36: 625-639.

Soubra, A.-H., Dias, D., Emeriault, F. and Kastner, R. (2008). "Three-dimensional face stability analysis of circular tunnels by a kinematical approach." Geocongress 2008, GSP N 179, ASCE, New Orleans/Louisiana: 894-901.

Youssef Abdel Massih, D.S., Soubra, A.-H., and Low, B.K. (2008). "Reliabilitybased analysis and design of strip footings against bearing capacity failure." $J$. of Geotech. \& Geoenvironmental Engrg., 134 (7): 917-928. 